\title{
Depletion of CDC5L inhibits bladder cancer tumorigenesis
}

Ziwei Zhang*, Weipu Mao*, Longsheng Wang*, Mengnan Liu, Wentao Zhang, Yuan Wu, Junfeng Zhang, Shiyu Mao, Jiang Geng ${ }^{\bowtie}$, Xudong Yao ${ }^{\bowtie}$

Department of Urology, Shanghai Tenth People's Hospital, Tongji University, Shanghai 200072, P. R. China.

* Contributed equally.

$\triangle$ Corresponding author: Prof. Xudong Yao, Tel: +8617602141290; E-mail: yaoxudong1967@163.com; Prof. Jiang Geng, Tel: +8613764285835; E-mail: gengjiangsh@sina.com Department of Urology, Shanghai Tenth People’s Hospital, Tongii University, Shanghai 200072, P. R. China.

(c) The author(s). This is an open access article distributed under the terms of the Creative Commons Attribution License (https://creativecommons.org/licenses/by/4.0/). See http://ivyspring.com/terms for full terms and conditions.

Received: 2019.01.06; Accepted: 2019.09.27; Published: 2020.01.01

\begin{abstract}
Cell division cycle 5-like (CDC5L) protein is a cell cycle regulator of the G2/M transition and has been reported to participate in the catalytic step of pre-messenger RNA (mRNA) splicing and DNA damage repair. Recently, CDC5L was also found to act as a candidate oncogene in osteosarcoma and cervical tumours. However, the role of CDC5L expression in bladder cancer remains unclear. Here, we analysed the expression and clinical significance of CDC5L in bladder cancer tissues. The expression of $C D C 5 \mathrm{~L}$ in fresh bladder cancer tissues and paraffin-embedded slices was evaluated by western blot and immunohistochemistry, respectively. We found that CDC5L was highly expressed in bladder cancer. The expression of CDC5L was significantly associated with bladder cancer pathology grade and $\mathrm{Ki} 67$ expression. Univariate and multivariate analyses showed that high CDC5L expression was an independent prognostic factor for the survival of bladder cancer patients. To determine whether CDC5L could regulate the proliferation of bladder cancer cells, we transfected bladder cancer cells with an interfering RNA targeting CDC5L and then investigated cell proliferation with a cell counting kit (CCK)-8, flow cytometry assays, colony formation and xenograft assay analyses. Our results indicate that knockdown of CDC5L inhibits proliferation of bladder cancer cells. In addition, reduced expression of CDC5L induced apoptosis of bladder cancer cells and inhibited their migration, invasion and EMT. These findings suggest that CDC5L might play an important role in bladder cancer and thus be a promising therapeutic target of bladder cancer.
\end{abstract}

Key words: CDC5L; bladder cancer; tumorigenesis.

\section{Introduction}

Bladder cancer $(\mathrm{BCa})$ is one of the most prevalent cancers of the genitourinary system and occurs more commonly in men than women [1]. Nearly three-quarters of bladder cancers are superficial [stages Ta, T1, carcinoma in situ (CIS)] at presentation which are now termed as non-muscle invasive bladder cancer (NMIBC) [2, 3].While the clinical treatment of bladder cancer has made great progress, the prognosis of $\mathrm{BCa}$ patients still remains unsatisfactory due to a high incidence of metastasis and invasion [4, 5].A number of gene mutations are closely related to the development of bladder cancer
[6].Therefore, research into the molecular aetiology may provide insights into the mechanism of the development of bladder cancer.

The Cell division cycle 5-like (CDC5L) protein has been well-known a significant similarity with the Schizosaccharomyces pombe cdc5 gene product, which is a cell cycle regulator essential for the G2/M transition [7-10]. CDC5L interacts with the cell cycle checkpoint protein ATR and activates effectors downstream of ATR, including Chk1 and Rad17. Interference with CDC5L inactivates the $S$ phase cell cycle checkpoint, thus increasing drug sensitivity [11]. 
In addition to affecting the cell cycle, CDC5L protein is also a member of the spliceosome complex and is involved in pre-mRNA splicing [12-14]. This suggests that CDC5L may be a very active protein. CDC5L also plays a key role in some human somatic tumours. CDC5L promotes the transcriptional activation of the hTERT promoter as an oncogene in colorectal cancer. It is reported that CDC5L possesses potential oncogenic activity in osteosarcoma and cervical tumours $[15,16]$. CDC5L is closely related to the mitotic stage of the cell cycle, so it is considered a potential target for tumour therapy [17, 18]. At present, there is no report on the role of CDC5L in bladder cancer and related mechanisms.

In this study, we demonstrated the expression and potential function of CDC5L in bladder cancer. These results suggest that CDC5L plays a crucial role in bladder cancer and may provide a new potential target for cancer therapy targets in bladder cancer.

\section{Materials and methods}

\section{Patients and tissue specimens}

Surgical specimens of bladder cancer tissues and paired normal bladder tissues were obtained from the Department of Urology, Shanghai Tenth People's Hospital, Tongji University (Shanghai, China) from January 2008 to December 2018. Following surgery, fresh tissue specimens were immediately snap-frozen in liquid nitrogen and stored at $-80{ }^{\circ} \mathrm{C}$ until further use. Written informed consent from all patients or their relatives and approval from the Ethics Committee of the Tenth People's Hospital was obtained.

\section{Tumour microarray and immunohistochemistry}

Paraffin sections were taken from patients with bladder cancer for tissue microarray. Human bladder cancer and adjacent normal tissues were fixed in cold $4 \%$ paraformaldehyde. Tumour-rich areas were board-certified by pathologists. After constructing the tissue microarray, the sections were stained for CDC5L. The pathological sections were assessed separately by at least two pathologists. Five fields of view were randomly selected from bladder cancer tissues and normal bladder tissues for histological scoring. Intensity was evaluated in comparison with the control and scored as follows: 0 (no staining), 1 (weak staining $=$ light yellow), 2 (moderate staining = yellow brown), and 3 (strong staining = brown). Scores representing the proportion of positively stained tumour were as follows: $0,<10 \% ; 1,11-25 \% ; 2$, $26-50 \% ; 3,51-75 \%$; and $4,>75 \%$. Scores from the two scales were combined, and we divided the expression of CDC5L into two grades: scores from $0-3$ were counted as low expression, while scores from $4-7$ were counted as overexpression.

\section{Cell lines and culture}

Human bladder cancer cell lines (T24, UMUC3 and J82) and human bladder epithelial permanent cell line SV-HUC-1 were purchased from the American Type Culture Collection (ATCC, Rockville, USA). SV-HUC-1 cells were maintained in F12K medium (Sigma-Aldrich, St. Louis, MO, USA). T24 and UMUC3 cells were cultured in RPMI-1640 medium (Gibco, Rockville, MD, USA), and J82 cells were grown in Dulbecco's modified Eagle's medium (Gibco). All cell culture media were added with 10\% foetal bovine serum (FBS; Gibco) and 1\% penicillin/streptomycin (HyClone, Logan, UT, USA). The cell lines were incubated at $37^{\circ} \mathrm{C}$ in a humidified atmosphere of $5 \% \mathrm{CO}_{2}$.

\section{RNA extraction and quantitative real-time PCR}

Total RNA was extracted from the cultured cells using TRIzol reagent (Invitrogen, Carlsbad, CA, USA). Purity and concentration of the RNA were determined using an ND-2000 spectrophotometer (Thermo Fisher Scientific, Inc., Carlsbad, CA, USA). The RNA was reverse-transcribed into cDNA using a cDNA synthesis kit (Takara, Kyoto, Japan), and qRT-PCR was performed using a SYBR Green PCR Kit (Takara Biotechnology, Dalian, China) with an ABI Prism 7500 Sequence Detection System (Applied Biosystems, Foster City, CA, USA). The primers for PCR analysis were as follows: CDC5L: 5'-TCTCT GAAG CTCC TCTC GGC-3' (forward) and 5'-CATC CTCG GTAT TCCT CCATACG-3' (reverse). $\beta$-actin: 5'-CCTGGCACCCAGCACAAT-3' (forward) and 5'-GGGCCGGACTCGTCATAC-3' (reverse). PCR conditions: $2 \mathrm{~min}$ at $95^{\circ} \mathrm{C}$, followed by 40 cycles of 15 sec at $95{ }^{\circ} \mathrm{C}$ and $30 \mathrm{sec}$ at $60{ }^{\circ} \mathrm{C}$. CDC5L mRNA expression was normalized to the $\beta$-actin mRNA. The relative expression levels of CDC5L were analysed using the $2^{-\Delta \Delta \mathrm{Ct}}$ method. Each sample was tested in triplicate.

\section{Cell transfection}

Three CDC5L siRNA sequences were designed by GenePharma(Shanghai, China) and finally we selected the one with the most obvious effect. The sequence of CDC5L siRNA was: 5'-CGCUGUUUGG UAUUUGGUAUU-3'. CDC5L siRNA (si-CDC5L) and negative control siRNA (si-NC) were all obtained from GenePharma. Lipofectamine 3000 (Invitrogen; Thermo Fisher Scientific, Inc., USA) was used for transfection, according to the manufacturer's 
instruction. The expression of CDC5L was examined at RNA and protein levels.

\section{Cell proliferation assay}

Cell proliferation rate was measured using a Cell Counting Kit-8 (CCK-8; Dojindo, Japan), according to the manufacturer's instructions. Simply speaking, the transfected cells were plated into 96-well plates at a density of $2 \times 10^{3}$ cells/well and grown for up to 5 days. Subsequently, CCK-8 reagent $(10 \mu \mathrm{l})$ was added to each well, and the cells were incubated at $37^{\circ} \mathrm{C}$ for 2 $\mathrm{h}$. The absorbance at $450 \mathrm{~nm}$ was determined using a microplate spectrophotometer (BioTek Instruments Inc., Winooski, VT, USA).

\section{Colony formation assay}

The transfected cells were seeded into 6-well plates at a density of 1,000 cells/well. After approximately 10 days from plating, the colonies were washed three times with PBS, fixed with methanol and stained with $0.1 \%$ crystal violet solution. Cell colonies $\geq 50$ cells were counted under a light microscope (Olympus Corporation, Tokyo, Japan). The experiment was performed at least three times.

\section{Xenograft assays in nude mice}

The lentivirus expressing the shRNA against CDC5L was produced by Lingke Biotechnology (Shanghai, China). T24 and UMUC3 cells were infected with LV-sh-CDC5L or LV-sh-NC and then selected using puromycin (Sigma-Aldrich). After generating stably transfected LV-sh-NC and LV-sh-CDC5L cell lines, cells were injected subcutaneously ( $3 \times 10^{6}$ in $0.1 \mathrm{~mL}$ of PBS) into mice. Tumour growth curves were measured weekly with a Vernier caliper from week 1-5. The tumour volume was calculated using the following formula: tumour volume $[\mathrm{mm} 3]=($ length $[\mathrm{mm}]) \times($ width $[\mathrm{mm}]) 2 \times 0.5$. After 5 weeks, the mice were sacrificed and tumour xenografts were resected and then stored at $-80{ }^{\circ} \mathrm{C}$ until further use. All animal studies were approved by the Institutional Animal Care and Use Committee of the Shanghai Tenth People's Hospital of Tongji University.

\section{Cell apoptosis assay}

Annexin-V/PI apoptosis detection kit (BD Pharmingen, Franklin Lakes, NJ, USA) was used to measure cell apoptosis, according to the manufacturer's instructions. First, the cells were collected, washed twice with cold PBS, resuspended in Annexin V-FITC and propidium iodide (PI), and stained in the dark at room temperature for $15 \mathrm{~min}$. Subsequently, apoptosis rates were analysed by flow cytometry (fluorescence-activated cell sorting, BD
Biosciences)

\section{Scratch wound healing assay}

For the scratch healing assay, the transfected cells were grown to full confluence in six-well plates. Then, the cells were wounded using a $200 \mu$ l sterile pipette tip, washed 3 times with PBS, and cultured in RPMI-1640 medium without FBS. The wounds were continuously photographed at a magnification of $x 5$ using an optical system microscope at 0 and 48 hours.

\section{Transwell invasion assay}

6.5-mm Transwell chambers with $8-\mu \mathrm{m}$ pore polycarbonate membrane inserts (BD Biosciences, San Jose, CA, USA) were used for invasion assays. A total of $5 \times 10^{4}$ cells/Transwell were plated in the top chamber of the Transwells in $200 \mu \mathrm{l}$ of FBS-free RPMI-1640 medium. The bottom chamber was filled with $500 \mu \mathrm{l}$ of cell culture medium containing $10 \%$ FBS. Furthermore, the insert of each Transwell was precoated with Matrigel (BD Biosciences, Franklin Lakes, NJ, USA), and the cells were allowed to invade. After about $24 \mathrm{~h}$, the cells remaining in the upper surface of the inserts were carefully wiped with a moist cotton swab, and the invading cells in the lower surface of the inserts were fixed with $70 \%$ ethanol for $15 \mathrm{~min}$ and stained with $0.1 \%$ crystal violet for $15 \mathrm{~min}$ and then observed under a light microscope (Olympus Corporation). Invasion abilities were defined as the number of invading cells. The experiments were repeated at least three times.

\section{Western blot assay}

Total proteins of tissues were extracted using ice-cold RIPA buffer (Sigma-Aldrich) containing a protease inhibitor. The protein concentration of lysates was measured using the BCA method. An equal amount of protein was separated by $10 \%$ sodium lauryl sulfate-polyacrylamide gels (SDS-PAGE) and transferred to nitrocellulose (NC) membranes. Afterward, the membranes were blocked with $5 \%$ non-fat milk in PBS for $1 \mathrm{~h}$ at room temperature and immunoblotted overnight with primary antibodies at $4{ }^{\circ} \mathrm{C}$. All primary antibodies were purchased from Abcam (Cambridge, Cambridge, UK). Then, the membranes were incubated with a fluorescence-conjugated secondary antibody (926-68072 or 926-32210, LI-COR Biosciences, Shanghai, China) for $1 \mathrm{~h}$ at room temperature. Subsequently, the membranes were washed in PBST three times and the protein bands visualized and quantified using the Odyssey twocolour infrared laser imaging system (LI-COR Biosciences, Lincoln, NE, USA). Three independent experiments were carried out for each assay. 


\section{Statistical analysis}

SPSS 16.0 software (SPSS Inc., Chicago, IL, USA) and GraphPad Prism 7 (GraphPad Software, Inc., La Jolla, CA, USA) were used to analyse the resulting data. Chi-square tests or Fisher's exact tests were used to assess the association between clinical pathological features and CDC5L expression, and Student's t test was used for comparisons between two groups. $\mathrm{P}<0.05$ was considered statistically significant.

\section{Results}

\section{CDC5L is upregulated in bladder cancer cells and tissues}

Previous studies have revealed that CDC5L plays an active role in the development of cancers. To determine the expression pattern of CDC5L in bladder cancer cells and tissues, western blot analysis and $\mathrm{qPCR}$ were performed to investigate the expression pattern of CDC5L in bladder cancer cells and tissues. The results suggest that the mRNA and protein expression of CDC5L were generally increased in the 3 bladder cell lines T24, UMUC3 and J82 compared with the human bladder epithelial permanent cell line SV-HUC-1(Fig. 1A and 1B). Consistently, CDC5L expression was also higher in bladder cancer tissues than in the matched normal bladder tissues (Fig. 1C).

A
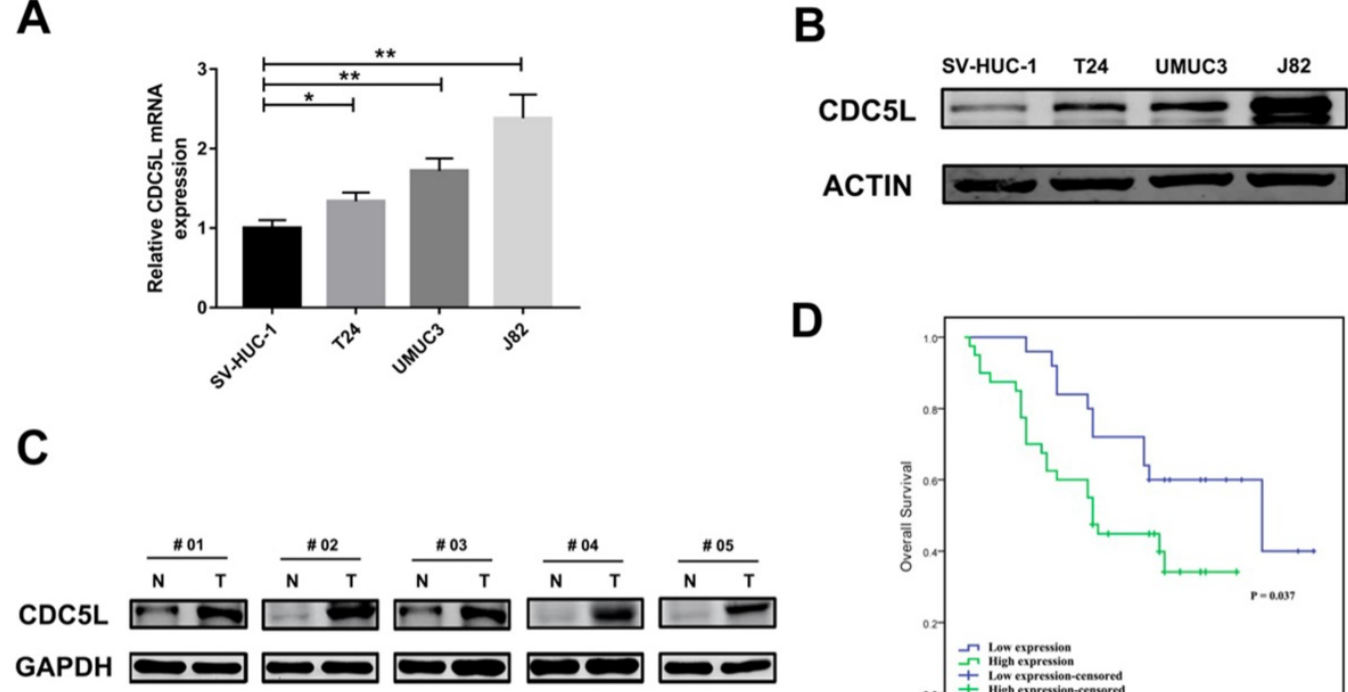

D

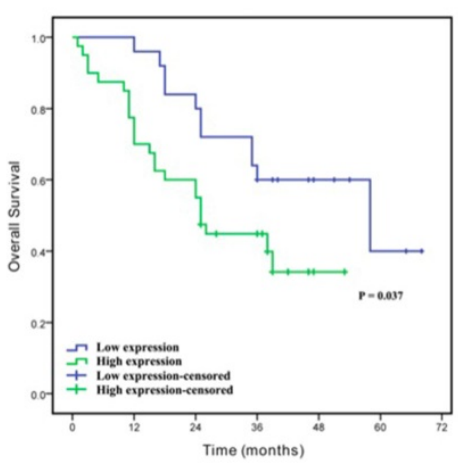

ACTIN
E
Patient 1

$10 \times$
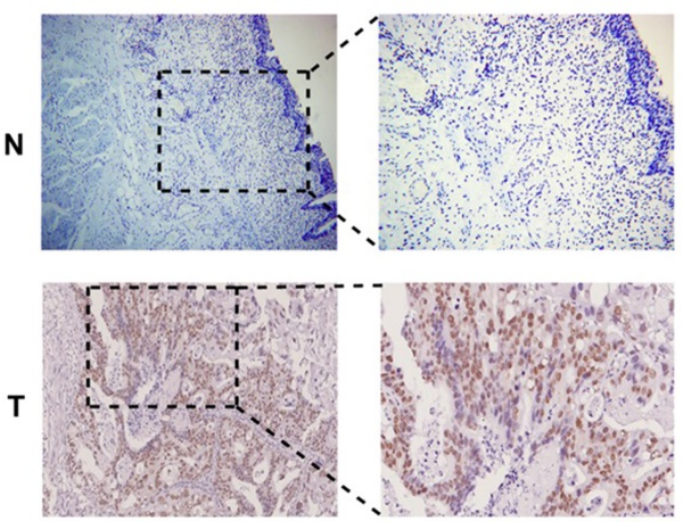

\begin{tabular}{ll}
\multicolumn{3}{c}{ Patient 1 } \\
\hline $10 \times \quad 20 \times$
\end{tabular}

Figure 1. CDC5L is upregulated in human bladder cancer cell lines and tissues. Protein (A) and mRNA (B) expression of CDC5L in bladder cancer cell lines (T24, UMUC3 and j82) and the immortalized human normal bladder epithelial cell line SV-HUC-1 determined by western blot and qRT-PCR, respectively. (C) protein levels of CDC5L in bladder cancer tissues $(\mathrm{T})$ and paired adjacent normal tissues $(\mathrm{N})$ detected by western blot. (D) Kaplan-Meier survival analysis of patients with bladder cancer according to CDC5L expression status. Kaplan-Meier postoperative survival curve for high CDC5L expression group (40 patients) and low CDC5L expression group (25 patients). Patients in the high-expression CDC5L group had significantly shorter overall survival $(\mathrm{P}<0.01)$. (E) Immunohistochemistry (IHC) staining of CDC5L in bladder cancer tissues (T), and in adjacent normal tissues (ANT). Representative images are shown. Scale bar $=200 \mu \mathrm{m}$ for $10 \times$ and $100 \mu \mathrm{m}$ for $20 \times$. 
A

CDC5L

ACTIN

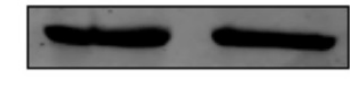

T24

\section{B}

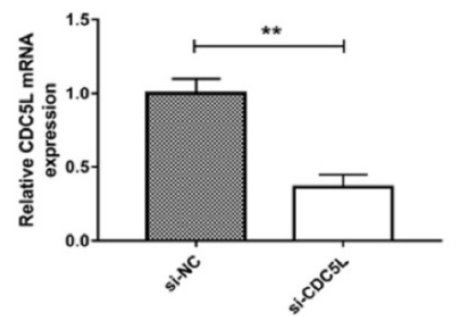

C
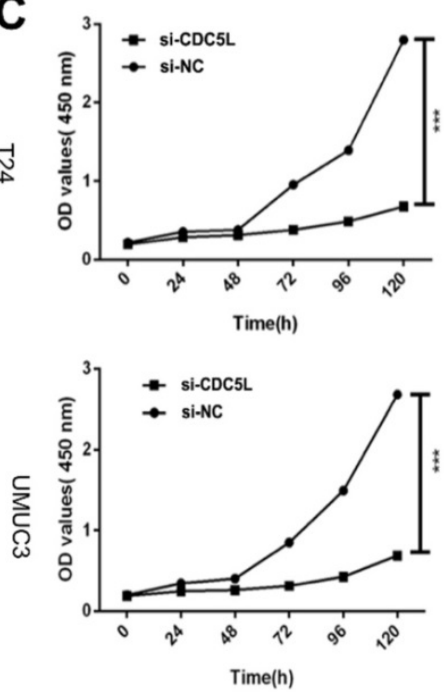

D

$\vec{\Upsilon}$
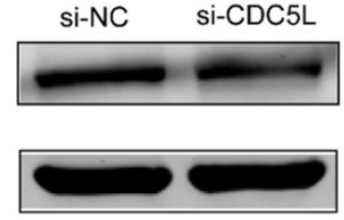

UMUC3
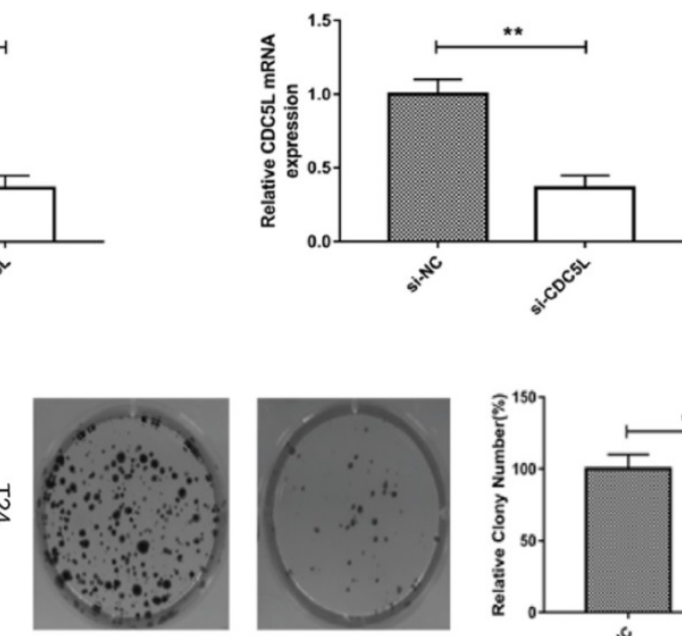

si-NC

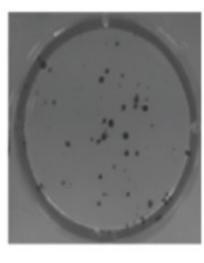

si-CDC5L
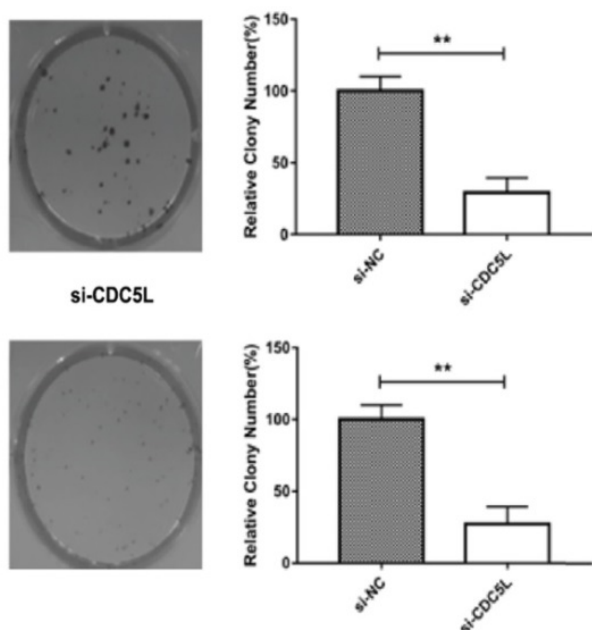

Figure 2. Knockdown of CDC5L inhibits cell growth of T24 and UMUC3 cells in vitro. (A) CDC5L protein expression was determined by western blot $72 \mathrm{~h}$ after transfection of si-CDC5L or si-NC. (B) CDC5L mRNA expression was detected by qRT-PCR $48 \mathrm{~h}$ after transfection of si-CDC5L or si-NC. CCK8 assay (C) and colony formation assay (D) were used to determine proliferation and colony-forming ability of si-CDC5L or si-NC-transfected T24 and UMUC3 cells. **P $<0.01$ vs. si-NC groups $(n=3)$.

We took sixty-five pairs of bladder cancer tissues and their adjacent normal tissue for tissue microarray. The stained tissue microarrays indicated that CDC5L was highly expressed in $61.5 \%(40 / 65)$ of bladder cancer tissues compared with $38.5 \%(25 / 65)$ of the matched normal tissues and the high expression of CDC5L between bladder tumour tissues and matched normal tissues was statistically significant (Fig. 1E). The clinicopathological factors of the 65 patients are listed in Table 1. As shown in Table 1, the expression of CDC5L was not related to age, gender, smoking status, BMI, diabetes status, or $\mathrm{T}$ stage but significantly correlated with histologic grade, $\mathrm{N}$ stage and $\mathrm{M}$ stage. Kaplan-Meier survival curves indicate that the high CDC5L expression was significantly associated with poorer overall survival and the difference was statistically significant (Fig. 1D). From these data, we infer that CDC5L might contribute to the progression of bladder cancer.

\section{Knockdown of CDC5L inhibited the growth of bladder cancer in vitro and in vivo}

To determine the biological effects of CDC5L on bladder cancer cell growth, T24 cells and UMUC3 cells were transfected with si-CDC5L or si-NC, respectively. The result revealed that the si-CDC5L was successfully transfected into the cells (Fig. 2A and 2B). Subsequently, CCK-8 and colony formation assays were used to study cell proliferation. The CCK-8 proliferation assay indicated that the proliferation of T24 and UMUC3 cells was suppressed markedly following infection with si-CDC5L (Fig. 2C). The colony formation assay revealed that knockdown of CDC5L significantly inhibited the proliferation of T24 cells and UMUC3 cells, when compared with the respective control (Fig. 2D). 
A

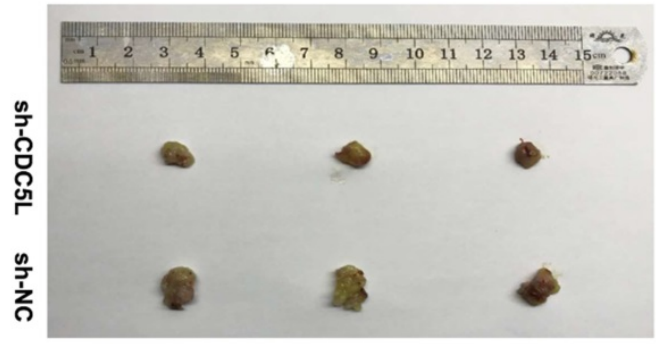

C

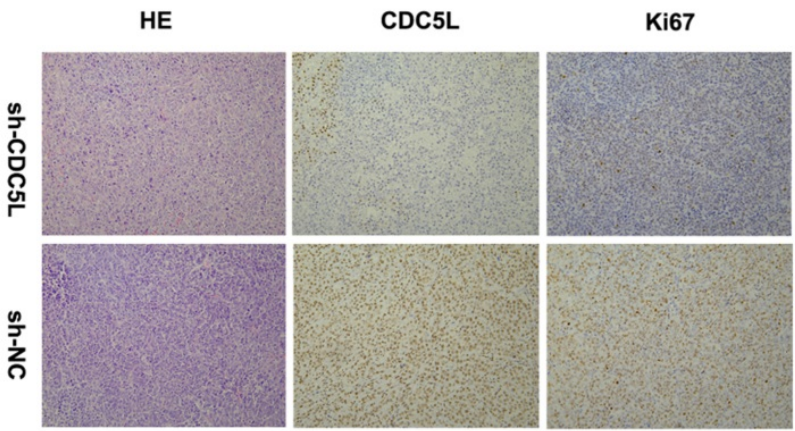

B

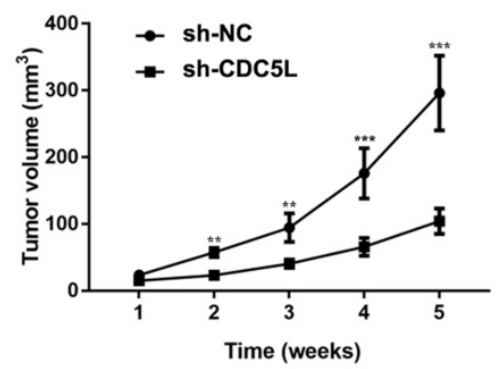

D

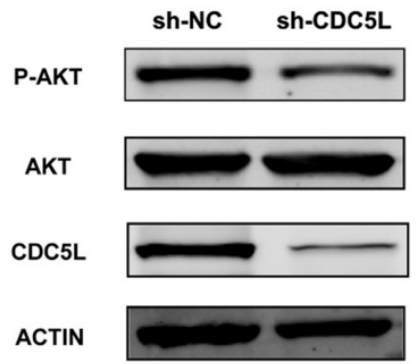

Figure 3. Knockdown of CDC5L inhibits xenograft tumour growth in vivo. Tumour growth curve (A) and tumour sizes (B) after T24 cells stably expressing low levels CDC5L or empty vectors were injected into 4-week-old nude mice. (C) Fewer Ki- $67^{+}$cells and lower CDC5L expression levels were detected by IHC in sh-CDC5L treated tumours. (D) Proteins were extracted from the tumour xenografts. CDC5L, p-AKT, and AKT expression was detected by western blotting. $\beta$-actin was used as an internal control. $* \mathrm{P}<0.05, * * \mathrm{P}<0.01$.

Table 1. Correlation of CDC5L expression with clinicopathological factors in 65 bladder cancer patients.

\begin{tabular}{|c|c|c|c|c|}
\hline \multirow[t]{2}{*}{ Characteristics } & \multirow[t]{2}{*}{ Total } & \multicolumn{2}{|c|}{ CDC5L expression } & \multirow[t]{2}{*}{$P$ value } \\
\hline & & Low & High & \\
\hline Total & 65 & 25 & 40 & \\
\hline Age (years) & & & & 0.136 \\
\hline$\leq 65$ & 34 & 16 & 18 & \\
\hline$>65$ & 31 & 9 & 22 & \\
\hline Gender & & & & 0.542 \\
\hline Male & 59 & 22 & 37 & \\
\hline Female & 6 & 3 & 3 & \\
\hline Smoking status & & & & 0.724 \\
\hline No & 33 & 12 & 21 & \\
\hline Yes & 32 & 13 & 19 & \\
\hline BMI & & & & 0.170 \\
\hline$\leq 24$ & 33 & 10 & 23 & \\
\hline$>24$ & 32 & 15 & 17 & \\
\hline Diabetes status & & & & 0.316 \\
\hline No & 51 & 18 & 33 & \\
\hline Yes & 14 & 7 & 7 & \\
\hline Histologic grade & & & & $0.023^{*}$ \\
\hline High & 8 & 6 & 2 & \\
\hline Low & 57 & 19 & 38 & \\
\hline Tumor stage & & & & 0.865 \\
\hline $\mathrm{T} 1-\mathrm{T} 2$ & 20 & 8 & 12 & \\
\hline T3-T4 & 45 & 17 & 28 & \\
\hline Lymphatic invasion & & & & $0.041^{*}$ \\
\hline Negative & 45 & 21 & 24 & \\
\hline Positive & 20 & 4 & 16 & \\
\hline Distant metastasis & & & & $0.047^{*}$ \\
\hline Negative & 60 & 21 & 39 & \\
\hline Positive & 5 & 4 & 1 & \\
\hline
\end{tabular}

Furthermore, to explore the function of CDC5L in vivo, nude mouse experiments were performed after establishing stable sh-CDC5L cells. After 5 weeks of tumour implantation, the tumour xenograft sizes of the sh-CDC5L group were dramatically reduced compared with the sh-NC group (Fig. 3A). Consistently, the sh-CDC5L group showed slower growth of the tumour xenografts compared with the sh-NC group (Fig. 3B). In addition, we detected the expression of Ki67 in the two groups immunohistologically. As shown in Fig. 3C, the tumour tissues from the CDC5L knockdown group showed lower CDC5L expression levels and carried fewer $\mathrm{Ki}-67^{+}$cells than in the control group, indicating a critical role for CDC5L in promoting bladder cancer growth in vivo.

To further explore the possible mechanism underlying the cell growth inhibition effect, we examined expression of PI3K/AKT family protein members by western blot. As illustrated in Fig. 3D, upon knockdown of CDC5L, the total phosphorylation level did not change but AKT phosphorylation was decreased. From the results above, we concluded that CDC5L plays a critical role in cell growth and proliferation partially through regulation of the PI3K/AKT signalling pathway. 
A
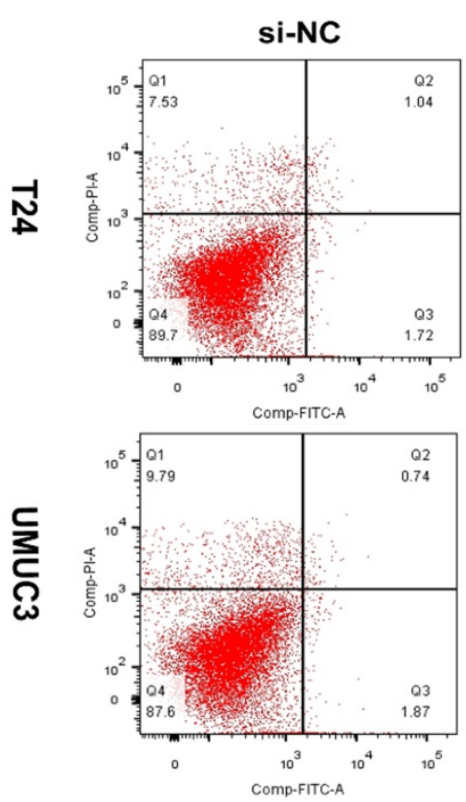

B

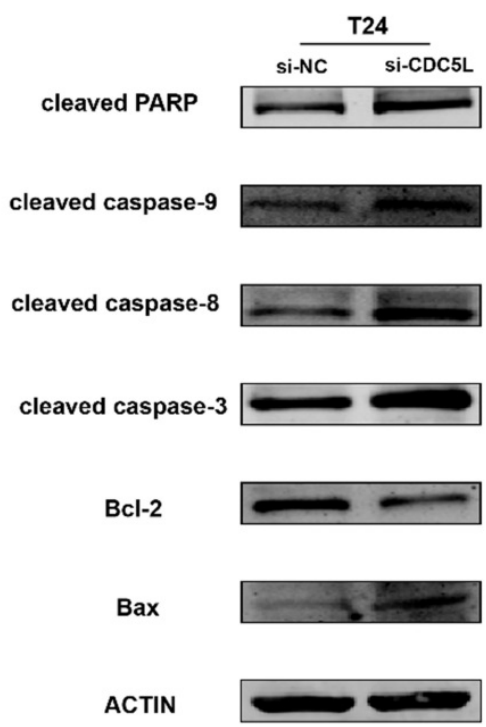

si-CDC5L
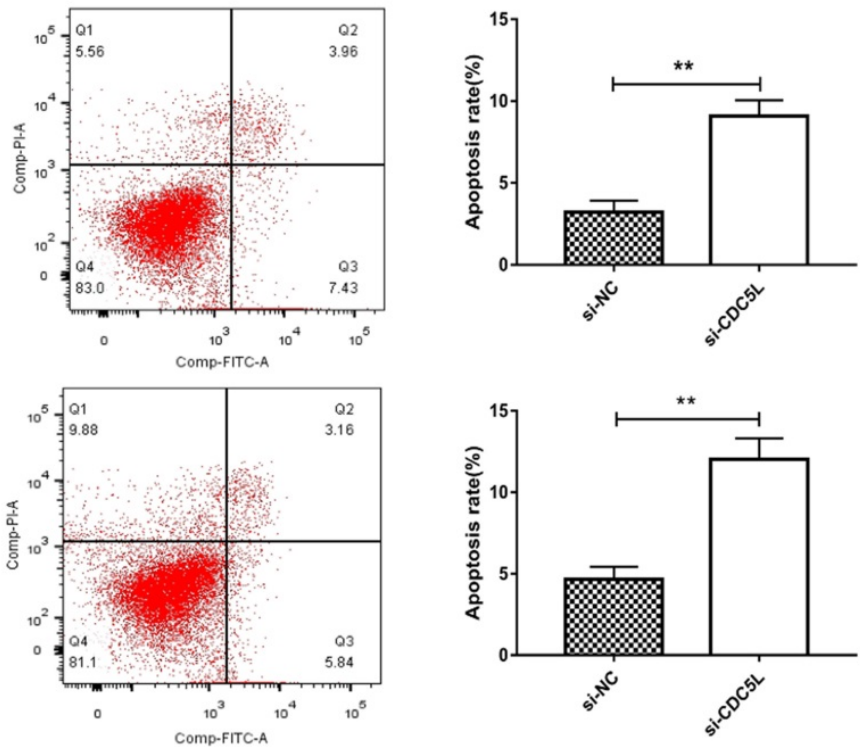

T24
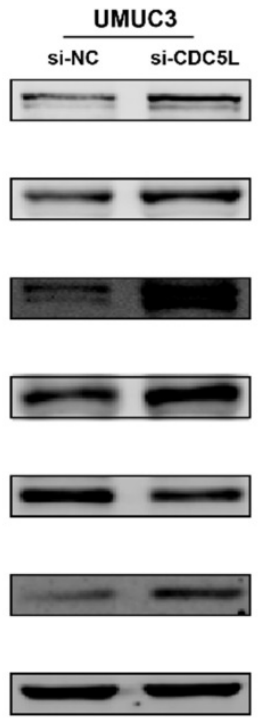

Figure 4. Reduction of CDC5L promoted bladder cancer cell apoptosis. (A) Flow cytometry assay for apoptotic rates in T24 and UMUC3 cells following CDC5L knockdown. (B)Representative cell apoptosis markers were determined by Western blot assay in T24 and UMUC3 cells, respectively. $\beta$-actin was used as an internal control. $* \mathrm{P}<0.05, * * \mathrm{P}<0.01(\mathrm{n}=3)$.

\section{Knockdown of CDC5L promoted bladder cancer cell apoptosis}

A previous study has shown that CDC5L is a regulator of mitotic progression [15], and so we predicted that CDC5L might have effects on bladder cancer cell apoptosis. Flow cytometry was used to analyse cell apoptosis. The percentage of cells at different phases revealed that CDC5L knockdown induced an increase in apoptosis of T24 and UMUC3 cells (Fig. 4A). To further understand the effect of CDC5L on bladder cancer cell apoptosis, the expression of several apoptosis related proteins was determined in bladder cancer cells after CDC5L knockdown. The protein expressions of the pro-apoptotic markers cleaved-PPAR, caspases 3, 8, and 9, and Bax were found to be upregulated while the protein expression of the anti-apoptotic marker Bcl-2 was found to be downregulated (Fig. 4B). Taken together, the current data indicated that CDC5L promotes the growth of bladder cancer due to the inhibition of apoptosis.

\section{Knockdown of CDC5L inhibits migration and invasion of bladder cancer cells}

To investigate the effect of CDC5L on the metastatic and invasion capacity of bladder cancer cells, cell scratch assay and Transwell assay were 
performed to examine migration and invasion abilities of both T24 and UMUC3 cells, respectively. It was observed that knockdown of CDC5L significantly reduced the tumour cell migration and invasion abilities compared to the control group (Fig. 5A and $5 B)$.

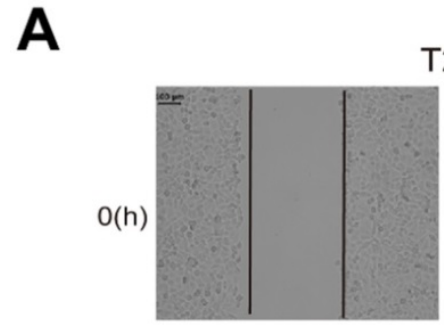

si-NC

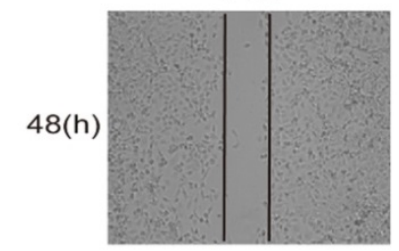

B

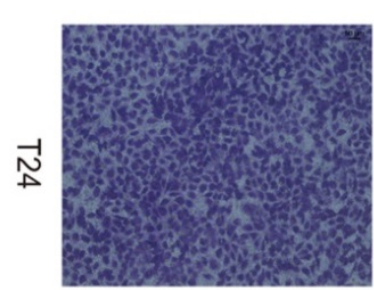

si-NC

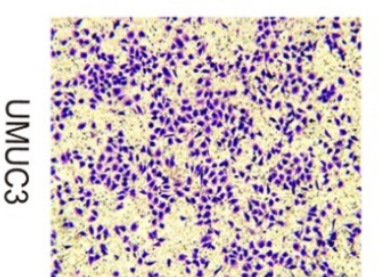

C

T24

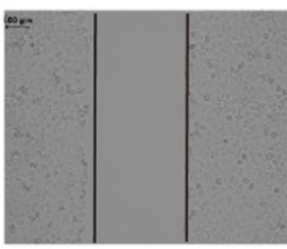
si-CDC5L
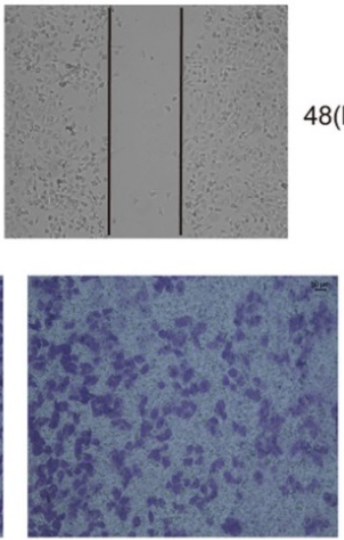

si-CDC5L

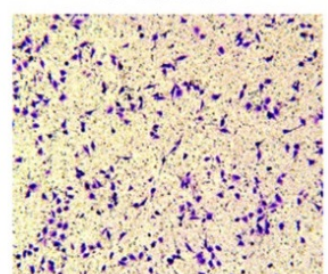

$48(h)$

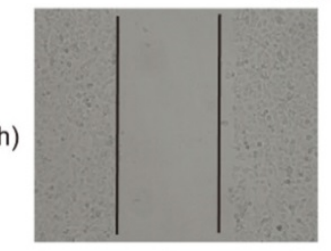

si-NC
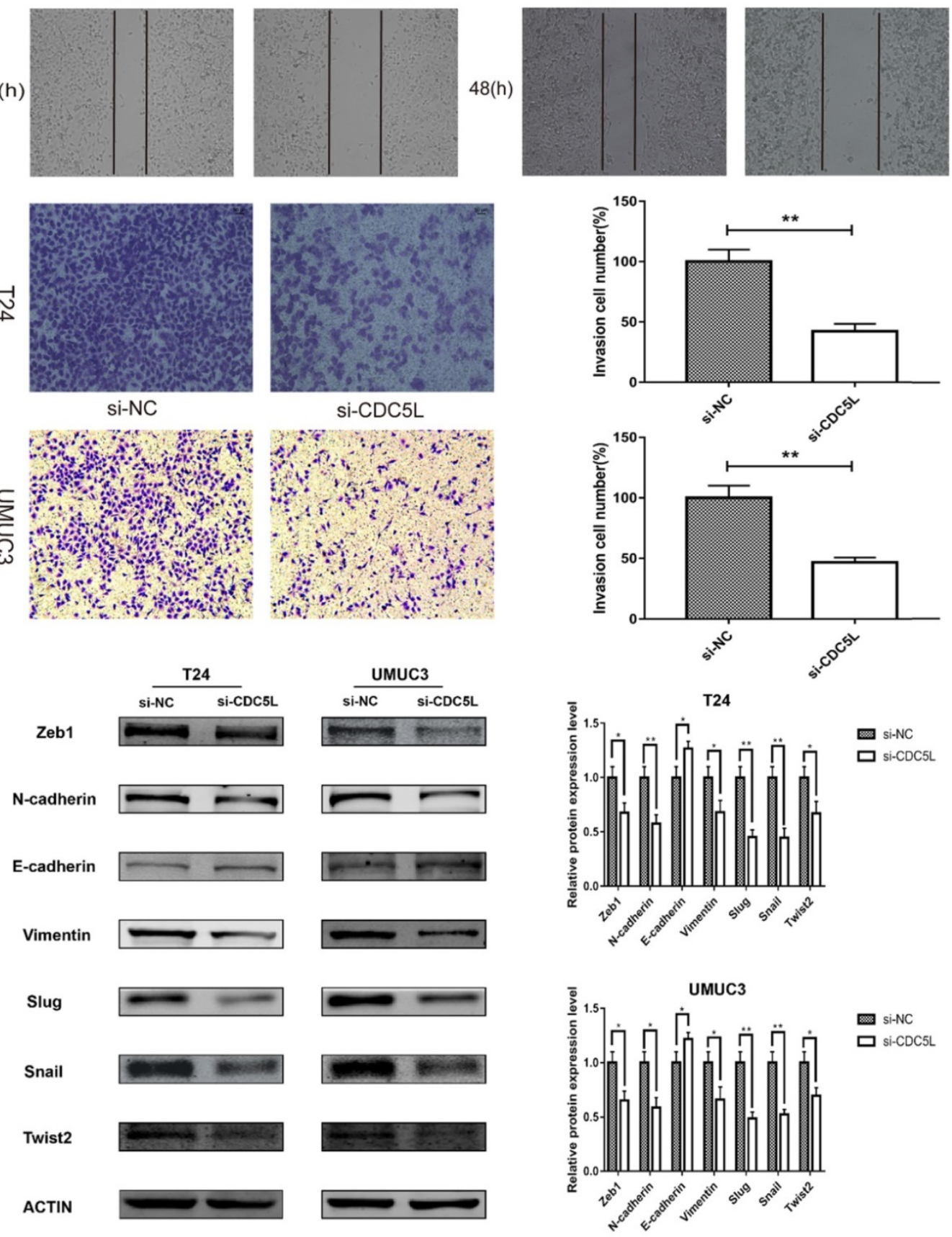

UMUC3

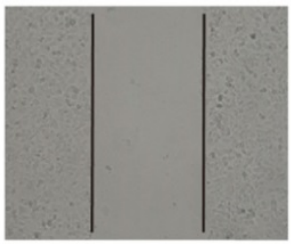

si-CDC5L

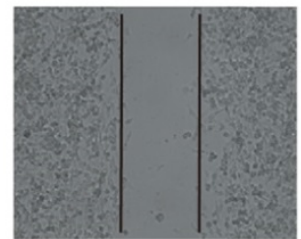

Figure 5. knockdown of CDC5L inhibits bladder cancer cell migration, invasion and EMT. Wound healing assay (A) and Transwell assay (B) were performed to examine migration and invasion of T24 and UMUC3 cells. (C) EMT-associated markers were measured by western blot analysis in T24 and UMUC3 cells, respectively. $\beta$-actin was used as an internal control. $* P<0.05, * * P<0.01 \quad(n=3)$. 


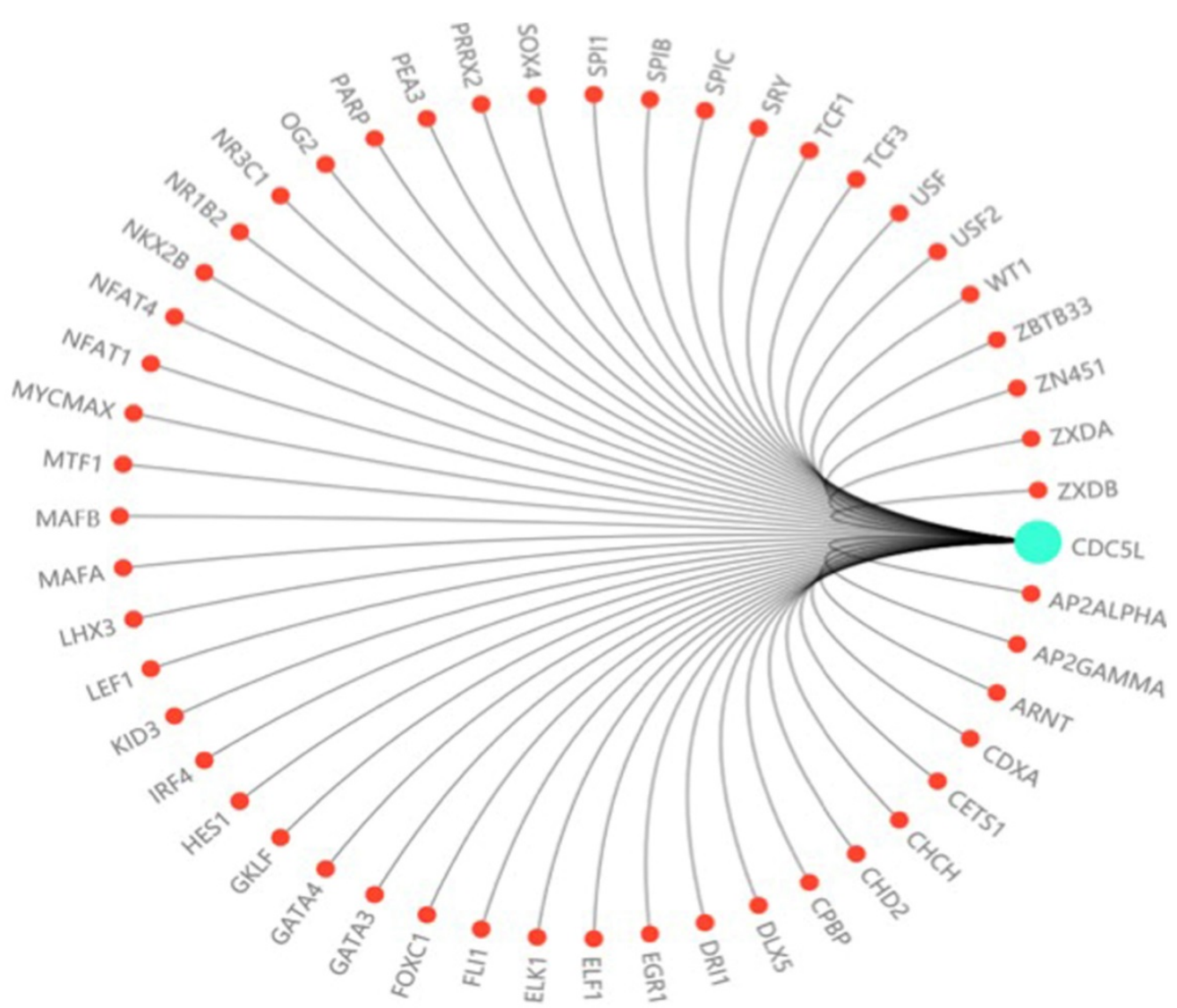

Figure 6. The prediction of transcription factor related to CDC5L. The highly recommended transcription factor related to $\mathrm{CDC} 5 \mathrm{~L}$ was obtained by integrating the information of the Transfac database, COSMIC database and dbSNP database.

\section{Knockdown of CDC5L inhibits bladder cancer epithelial-mesenchymal transition (EMT)}

EMT is a cellular program that is known to confer on cancer cells increased tumour-initiating and metastatic potential [19]. The expression of several EMT- related markers was also affected by the knockdown of CDC5L. In this study, knockdown of CDC5L significantly reduced the protein expression of mesenchymal markers $\mathrm{N}$-cadherin and vimentin as well as the transcription factors Zeb1, Slug, Snail and Twist2 compared to the si-NC group. Conversely, the expression level of the epithelial marker E-cadherin was significantly upregulated (Fig. 5C). Therefore, our results suggest CDC5L contributes to promotion of EMT in bladder cancer progression.

\section{Discussion}

With the development of molecular biology, the application of genetic diagnostic techniques promises to change bladder cancer clinical practice in the future [20]. Great progress has been made in the management of patients with bladder cancer over the past few years; however, the underlying molecular mechanisms of this disease remain to be studied. Here, we found that CDC5L was highly expressed in bladder cancer and played an important role in the tumorigenesis of bladder cancer.

Cell cycle regulation plays a critical role in malignant transformation and in the development of resistance to chemotherapy. Thus, targeting the cell cycle is increasingly considered an approach to cancer therapy [21, 22]. A previous study has shown that CDC5L is a regulator of mitotic progression, as depletion of CDC5L inhibits mitotic progression and eventually leads to mitotic catastrophe [15]. CDC5L was involved in the progression of hepatocellular carcinoma and was significantly associated with multiple clinicopathological factors. Depletion of CDC5L induced cell cycle arrest at the G2/M phase and reduced cell growth of hepatocellular carcinoma [23]. CDC5L has a similar effect in gliomas. Downregulation of CDC5L results in the apoptosis of glioma cells [24]. Therefore, CDC5L is related to cell size and cell proliferation. Amplification of chromosomal bands 6p12-p21 appeared to be significant in the pathogenesis of osteosarcoma, and CDC5L is found to be the most likely candidate oncogene for the 6p12-p21 amplicon [25].CDC5L was highly expressed in colon cancer cells and was correlated with cell growth and proliferation partially by regulating the PI3K/AKT signalling pathway. 
Consistently, we found that depletion of CDC5L in bladder cancer cells induced a decrease in AKT phosphorylation, but the total phosphorylation level did not change.

In this study, we investigated the effect of CDC5L on bladder cancer. CDC5L was highly expressed in bladder cancer and the expression of CDC5L was associated with multiple clinicopathological factors. Knockdown of CDC5L inhibited the growth of bladder cancer by promoting cell apoptosis. CDC5L also promoted cell proliferation by regulating PI3K/AKT signalling. Animal model assays suggested that CDC5L promotes cell proliferation not only in vitro but also in vivo. And CDC5L has a positive effect on the tumour cell migration and invasion abilities by cell scratch assay and Transwell assay. We have further confirmed that CDC5L plays an important role in the metastasis of bladder cancer through the validation of clinical patient datas. In addition, we demonstrated for the first time that CDC5L has a significant effect on bladder cancer cell EMT, which closely relates to bladder cancer cell metastasis. Moreover, further study is needed to fully understand the underlying mechanism of CDC5L in the development of bladder cancer. For example, according to the predicted score of the Transfac database and the information of the COSMIC database and dbSNP database, we predicted dozens of highly recommended transcription factors related to CDC5L (Fig. 6). These transcription factors may regard as an important way to study the upstream signalling pathway of this gene.

\section{Conclusions}

In summary, these findings suggest that CDC5L may play important roles in bladder cancer and we have confirmed that CDC5L contribute to the tumorigenesis of bladder cancer in vitro and in vivo. Therefore, CDC5L may serve as a novel target for bladder cancer therapy in the future.

\section{Acknowledgements}

This work was supported in part by a grant from the National Natural Science Foundation of China (\#81472389).

\section{Author's contributions}

$Z Z$, ML, JG and XY were involved in the study conception and design. $\mathrm{YW}, \mathrm{JZ}$ and $\mathrm{SM}$ collected the data. WM, LW, WZ and ZZ analyzed the data. ZZ and WM wrote the manuscript.

\section{Ethics approval and informed consent}

All patients' sample usage protocol was approved by the Ethics Committees of Shanghai
Tenth People's Hospital. And written informed consent was obtained from all patients or their relatives.

\section{Patient consent for publication}

All patients and their relatives consent to the publication.

\section{Competing Interests}

The authors have declared that no competing interest exists.

\section{References}

1. Knowles MA, Hurst CD. Molecular biology of bladder cancer: new insights into pathogenesis and clinical diversity. Nature Reviews Cancer. 2015; 15: 25.

2. Burger M, Catto JW, Dalbagni G, Grossman HB, Herr H, Karakiewicz P, et al. Epidemiology and risk factors of urothelial bladder cancer. European Urology. 2013; 63: 234-41.

3. Ranzi AD, Da SJ, Graziottin TM, Annels N, Bica CG. Immunohistochemistry Biomarkers in Nonmuscle Invasive Bladder Cancer. Applied Immunohistochemistry \& Molecular Morphology Aimm. 2015; 25: 1.

4. Chou R, Selph SS, Buckley DI, Gustafson KS, Griffin JC, Grusing SE, et al. Treatment of muscle-invasive bladder cancer: A systematic review. Cancer. 2016; 122: 842-51.

5. Powles T, Eder JP, Fine GD, Braiteh FS, Loriot Y, Cruz C, et al. MPDL3280A (anti-PD-L1) treatment leads to clinical activity in metastatic bladder cancer. Nature. 2014; 194: 558-62.

6. Chavan S, Bray F, Lortet-Tieulent J, Goodman M, Jemal A. International variations in bladder cancer incidence and mortality. European Urology. 2014; 66: 59-73.

7. Bernstein HS, Coughlin SR. A mammalian homolog of fission yeast Cdc5 regulates G2 progression and mitotic entry. Journal of Biological Chemistry. 1998; 273: 4666-71.

8. Gräub R, Lancero H, Pedersen A, Chu M, Padmanabhan K, Xu XQ, et al. Cell cycle-dependent phosphorylation of human $\mathrm{CDC} 5$ regulates RNA processing. Cell Cycle. 2008; 7: 1795-803.

9. Nasmyth K, Nurse P. Cell division cycle mutants altered in DNA replication and mitosis in the fission yeast Schizosaccharomyces pombe. Molecular \& General Genetics Mgg. 1981; 182: 119-24

10. Grieco M, Cerrato A, Santoro M, Fusco A, Melillo RM, Vecchio G. Cloning and characterization of H4 (D10S170), a gene involved in RET rearrangements in vivo. Oncogene. 1994; 9: 2531-5.

11. Zhang N, Kaur R, Akhter S, Legerski RJ. Cdc5L interacts with ATR and is required for the S-phase cell-cycle checkpoint. Embo Reports. 2009; 10: 1029.

12. Neubauer G, King A, Rappsilber J, Calvio C, Watson M, Ajuh P, et al. Mass spectrometry and EST-database searching allows characterization of the multi-protein spliceosome complex. Nature Genetics. 1998; 20: 46-50.

13. Burns CG, Ohi R, Krainer AR, Gould KL. Evidence that Myb-related CDC5 proteins are required for pre-mRNA splicing. Proceedings of the National Academy of Sciences of the United States of America. 1999; 96: 13789-94.

14. Ajuh P, Kuster B, Panov K, Zomerdijk JCBM, Mann M, Lamond AI. Functional analysis of the human CDC5L complex and identification of its components by mass spectrometry. Embo Journal. 2014; 19: 6569-81.

15. Mu R, Wang YB, Wu M, Yang Y, Song W, Li T, et al. Depletion of pre-mRNA splicing factor $\mathrm{Cdc} 5 \mathrm{~L}$ inhibits mitotic progression and triggers mitotic catastrophe. Cell Death \& Disease. 2014; 5: e1151.

16. Lu XY, Lu Y, Zhao YJ, Jaeweon K, Kang J, Li XN, et al. Cell Cycle Regulator Gene CDC5L, a Potential Target for 6p12-p21 Amplicon in Osteosarcoma. Molecular Cancer Research. 2008; 6: 937-46.

17. Geiss GK, Bumgarner RE, Brian B, Timothy D, Naeem D, Dunaway DL, et al. Direct multiplexed measurement of gene expression with color-coded probe pairs. Nature Biotechnology. 2008; 26: 317-25.

18. Nathan SS, Pereira BP, Zhou YF, Gupta A, Dombrowski C, Soong R, et al. Elevated expression of Runx2 as a key parameter in the etiology of osteosarcoma. Molecular Biology Reports. 2009; 36: 153-8.

19. $\mathrm{Wu} Y$, Zhou BP. New insights of epithelial-mesenchymal transition in cancer metastasis. Acta Biochimica Et Biophysica Sinica. 2010; 40: 643-50.

20. Costello JC, Dan T. Decade in review-bladder cancer: International progress: from cytology to genomics. Nature Reviews Urology. 2014; 11: 609-10.

21. Uzma A, Witkiewicz AK, Turner NC, Knudsen ES. The history and future of targeting cyclin-dependent kinases in cancer therapy. Nature Reviews Drug Discovery. 2015; 14: 130-46.

22. Schwartz GK, Shah MA. Targeting the cell cycle: a new approach to cancer therapy. Journal of Clinical Oncology. 2005; 23: 9408-21.

23. Qiu H, Zhang X, Ni W, Shi W, Fan H, Xu J, et al. Expression and Clinical Role of Cdc5L as a Novel Cell Cycle Protein in Hepatocellular Carcinoma. Digestive Diseases \& Sciences. 2016; 61: 795-805. 
24. Chen W, Li Z, Yan W, Jie S, Wang D, Fan S, et al. Expression of CDC5L is associated with tumor progression in gliomas. Tumour Biology the Journal of the International Society for Oncodevelopmental Biology \& Medicine. 2015; 37: 1-11.

25. Lau CC, Harris CPLu XY, Perlaky L, Gogineni S, Chintagumpala M, Hicks J, et al. Frequent amplification and rearrangement of chromosomal bands 6p12-p21 and 17p11.2 in osteosarcoma. Genes Chromosomes Cancer. 2004; 39: 11-21. 